\title{
RENDIMIENTO Y EFICIENCIA DE DISTINTAS TÉCNICAS DE CONTROL EN UN CALEFÓN ELÉCTRICO
}

\author{
PERFORMANCE AND EFFICIENCY OF \\ DIFFERENT CONTROL TECHNIQUES IN AN \\ ELECTRICAL HEATER
}

\author{
Gabriel V. Moreano ${ }^{1, *}$, César D. Arregui ${ }^{1}$, Camilo G. Tenesaca ${ }^{1}$, Henry J. Pilco ${ }^{1}$

\section{Resumen}

\section{Abstract}

El calentamiento de agua en el sector residencial ecuatoriano se ha convertido en un espacio de investigación y desarrollo, debido al intento de mitigar el gasto corriente de las personas y a la vez contribuir de manera activa a los procesos de eficiencia energética que van tomando fuerza en el país. En el presente documento se muestra un análisis comparativo entre diferentes maneras de controlar la temperatura del agua para un sistema residencial utilizando un calentador eléctrico; se analizó la respuesta de un conversor $\mathrm{AC} / \mathrm{AC}$ de control de fase directa que permite retrasar el ángulo de disparo de la onda de corriente alterna y la respuesta del control ON/OFF que activa o desactiva el calentador durante un número preestablecido de semiciclos de corriente alterna. Para las pruebas se instaló un prototipo de calentador eléctrico con un serpentín de 14 metros a base de resistencias eléctricas; con las respuestas de temperatura que se generan de cada conversor se procedió a identificar la función de transferencia de cada sistema ya que ambos difieren en su técnica de transmisión de calor y a la vez en su modelo matemático. Posteriormente se procedió a sintonizar un controlador PID para cada sistema, obteniendo buenos resultados de respuesta de temperatura en ambos casos, pero solo uno resultó eficiente en ahorro energético.

Palabras clave: control, PID, conversores AC/AC, temperatura, agua, eficiencia
Water heating in the Ecuadorian residential sector has become a space for research and development, due to the attempt to mitigate the current spending of people and at the same time contribute actively to the energy efficiency processes that are gaining strength in the country. This document shows a comparative analysis between different ways of controlling the water temperature for a residential system using an electric heater; the response of a direct phase control AC / AC converter was analyzed, which allows to delay the firing angle of the $\mathrm{AC}$ wave and the response of the ON / OFF control that activates or deactivates the heater during a pre-established number of half-cycles of current alternating, for the tests a prototype of electric heater was implemented with a coil of 14 meters based on electrical resistances, with the temperature responses that are generated from each converter, we proceeded to identify the transfer function of each system since both differ in its heat transmission technique and at the same time in its mathematical model, a PID controller was then tuned for each system, obtaining good results of temperature response in both cases but only one was efficient in energy saving.

Keywords: control, PID, AC / AC converters, temperature, water, efficiency

\footnotetext{
$\overline{1, *}$ Grupo de Investigación y Mantenimiento (GIMAN), Escuela Superior Politécnica de Chimborazo (ESPOCH), Ecuador, Autor para correspondencia : gabriel.moreano@espoch.edu.ec. (D) http://orcid.org/0000-0002-9331-8223,

(D) http://orcid.org/0000-0002-6055-916X, (D) http://orcid.org/0000-0001-8254-7998,

(D) http://orcid.org/0000-0002-0391-9755.
}

Recibido: 15-05-2019, aprobado tras revisión: 21-06-2019

Forma sugerida de citación: Moreano, G. V.; Arregui, C. D.; Tenesaca, C. G. y Pilco, H. J. (2019). «Rendimiento y eficiencia de distintas técnicas de control en un calefón eléctrico». INGENIUS. N. ${ }^{\circ} 22$, (julio-diciembre). pp. 90-99. DOI: https://doi.org/10.17163/ings.n22.2019.09. 


\section{Introducción}

La fuente de energía más empleada en el Ecuador para el calentamiento de agua y cocción de alimentos es el gas licuado de petróleo o GLP, pero a partir del cambio de la matriz energética este tipo de dispositivos presentan un impuesto del $100 \%$ sobre su valor comercial según el artículo 82 del reglamento de régimen tributario [1], razón por la cual se opta por promover el uso de otro tipo de calentadores como alternativas al excesivo incremento en el costo de los calentadores de GLP.

En [2] y [3] se muestran patentes originarias de lo que es un calefón de gas, se observa que se trata de dispositivos con bastante tiempo en el mercado. En [4] se aprecia el método de operación de estos dispositivos mientras que en [5] se observa un estudio completo sobre la aplicación de técnicas de control para este tipo de calentadores. Una de las principales dificultades que presentan estos equipos es la alta contaminación que ocasionan como lo menciona el autor en [6] sobre un estudio realizado en la ciudad de Loja - Ecuador; este problema genera grandes inconvenientes en la salud de las personas. Un análisis pertinente sobre estas afectaciones en la salud lo podemos encontrar en [7].

En otro aspecto, los calefones que usan GLP proporcionan un mayor o menor costo de funcionamiento dependiendo del valor que tenga el GLP en cada región; en las referencias [8-10] se muestran estudios comparativos entre el uso de un calefón de GLP y otras alternativas de calentamiento de agua residencial como calentadores solares o calentadores de gas natural. El beneficio en el uso de uno u otro sistema depende específicamente de los precios de cada fuente de energía, teniendo en cuenta que esto dependerá de los recursos de cada nación; existen países con enormes fuentes hídricas como Ecuador y países donde se dispone con mayor facilidad de derivados del petróleo, mientras en ciertas naciones desarrolladas como Alemania se ha optado por regular el autoconsumo donde más de un millón de viviendas incorporan paneles solares. Recientemente España también adoptó este camino donde hace poco se eliminó el llamado impuesto al sol [11], con lo que el Gobierno espera incremente el autoconsumo beneficiando los recursos nacionales.

Una alternativa válida para suplir a los sistemas de calentamiento de agua que utilicen GLP por seguridad y reducción en la generación de gases contaminantes es la utilización de calentadores eléctricos. Los calentadores eléctricos desempeñan un papel importante en el sistema de calefacción según [12] donde se indica que en un calentador eléctrico el $99 \%$ de la energía que consume se convierte en calor, por lo tanto, su potencia eléctrica es casi la misma que su potencia térmica. Varios autores muestran en [13] la eficiencia que puede tener un calefactor eléctrico asociado a una técnica de control a fin de regular la temperatura.
En [14] los autores realizan un resumen sumamente completo de algunos tipos de calefones eléctricos explicando distintos tipos de control de temperatura.

Actualmente, el defecto más importante que se encuentra en los calentadores eléctricos es el alto consumo energético que estos presentan, debido a que para calentar cierto caudal de agua se necesita una alta potencia eléctrica, en casos generales esta potencia es generada por una niquelina que permanecerá conectada a la red eléctrica al $100 \%$ de su potencia mientras un grifo de agua esté abierto, esto representa un rubro elevado en el pago de la energía eléctrica y, más aún, si este dispositivo permanece encendido varias horas al día.

La propuesta que se presenta en este trabajo es la combinación de la electrónica de potencia con la teoría de sistemas de control automático para disponer de un prototipo que regule la potencia que se disipará en una niquelina eléctrica reduciendo así el consumo energético y también mejorando la respuesta de temperatura del agua.

Para observar el tipo de respuesta tanto en eficiencia como en respuesta de temperatura se pusieron en funcionamiento dos conversores estáticos $\mathrm{AC} / \mathrm{AC}$ que comandarán el encendido o apagado de cuatro niquelinas eléctricas, el conversor por ciclo integral y el conversor de control por fase directa; se consulta en [15] el beneficio directo: ambos tipos de conversores se pueden implementar con el mismo circuito electrónico de potencia (ver Figura 1).

El microprocesador utilizado como dispositivo de control del sistema fue una de las versiones de Arduino que brinden cierta versatilidad a la hora de su utilización. En [16] se indica que mediante la utilización de una placa Arduino Mega 2560 se puede diseñar un grifo automático que permite tener una temperatura del fluido elevada (alrededor de $40{ }^{\circ} \mathrm{C}$ ) sin problemas de ruido electromagnético ni interferencias.

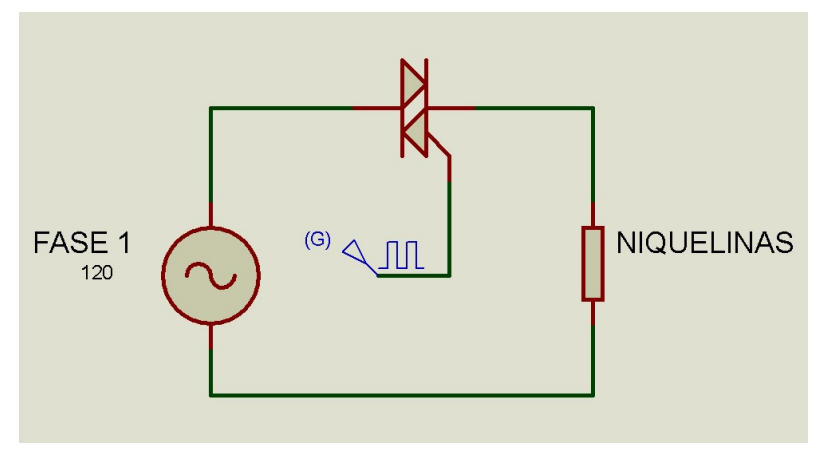

Figura 1. Esquema de simulación del conversor AC/AC.

En [17] se analiza el principio de funcionamiento de Arduino, además del interés de las personas por utilizar esta placa debido a que emplea una versión simplificada de $\mathrm{C}++$ de fácil utilización. 
Según [18] Arduino tiene memoria, capacidad de procesamiento autónomo, compiladores de lenguajes de programación como $\mathrm{C}$ y puertos físicos para interconectar con dispositivos tales que brindan cierta estabilidad y confianza en su utilización.

En [19] se menciona que, por su bajo costo, los microcontroladores Arduino se usan en aplicaciones de ingeniería que comúnmente involucran instrumentación, monitoreo de máquinas y estructuras y control de sistemas mecánicos.

Para la implementación del sistema de control PID se necesita medir las variables físicas a controlar en este caso la temperatura, para ellos se usa un termistor NTC de un vehículo; este tipo de sensores son fiables ya que son diseñados para trabajar en contacto con el agua, además, son sumamente económicos. En [20] los autores presentan una explicación sencilla del funcionamiento de los termistores NTC y como convierten una señal de temperatura en otra eléctrica.

En [21] se menciona que para el acondicionamiento del termistor se debe instalar un circuito que contiene una fuente de alimentación, un termistor y un resistor, generando un divisor de voltaje que es leído por el microprocesador. En el caso del sensor de temperatura este mantendrá su curva exponencial característica por lo que se deberá adquirir el valor con la ecuación del termistor en el microprocesador.

En [22] se muestra un análisis del circuito de control por ángulo de fase, el funcionamiento de este tipo de control se basa en un ángulo de retardo de encendido de las niquelinas, tanto en el semiciclo positivo como en el negativo de la onda sinusoidal eléctrica comercial. Así pues, variando el ángulo de disparo, se controla la potencia en cada niquelina y se regula la trasferencia de temperatura; en [23] se menciona que el interruptor o elemento del circuito que controla el encendido y apagado de las niquelinas puede ser un TRIAC o un conjunto de 2 SCR en conectados en antiparalelo.

En [24] los autores analizan el funcionamiento del control ON/OFF o llamado control por ciclo integral; su funcionamiento se basa en encender y apagar una carga varias veces en un periodo de tiempo de tal manera que se tenga encendidas las niquelinas un número conocido de ciclos, estos pueden cambiar según las necesidades de temperatura.

En [25] se explica una de las técnicas para la sintonización de reguladores PID. En este trabajo se optó por utilizar el modelo ARMAX de Matlab para obtener la función de trasferencia de cada sistema y posteriormente sintonizar dos controladores efectivos mediante el mismo software, el sintonizador automático de Matlab realiza un análisis iterativo para encontrar los mejores parámetros proporcional $(\mathrm{Kp})$, integral (Ki) y derivativo (Kd) del regulador PID. En [26] el autor menciona que a pesar de que se han desarrollado nuevas técnicas de control mucho más robustas, el control proporcional integral derivativo (PID) es la estrategia de control más usada en las aplicaciones industriales; se estima que más del $90 \%$ de los lazos de control utilizan un controlador PID, dado que es una estrategia simple, efectiva y no requiere una gran fundamentación teórica para su utilización en los procesos cotidianos.

\section{Métodos}

El objetivo principal del presente trabajo fue desarrollar un prototipo capaz de calentar agua de un estado cotidiano en la Sierra ecuatoriana, es decir, agua a una temperatura de unos $17{ }^{\circ} \mathrm{C}$ hasta una temperatura de $40{ }^{\circ} \mathrm{C}$ y mediante el prototipo poder evidenciar qué técnica de control genera mejor rendimiento tanto en el mantenimiento de la temperatura como en la eficiencia energética.

El presente prototipo está construido por un serpentín de 14 metros de longitud, construido a base de 4 niquelinas eléctricas, estas se conectan o desconectan de la red eléctrica comercial de $220 \mathrm{VAC}$, según las técnicas de control lo indiquen.

El control por ciclo integral varía el número de ciclos que permanecen encendidas las niquelinas con la ayuda del regulador PID. Entendiendo como un ciclo a un periodo completo de la onda sinusoidal de la red eléctrica comercial.

Mientras que el control por ángulo de fase varía el ángulo de disparo con que se activan las niquelinas. Si el periodo de la onda sinusoidal es de 16,66 ms, cada semiciclo tendrá un tiempo de $8,33 \mathrm{~ms}$, entonces el ángulo de disparo podrá variar entre 0 y $8,33 \mathrm{~ms}$ para encender o apagar las niquelinas según los requerimientos de temperatura.

\subsection{Potencia de las niquelinas}

La potencia se dimensiona considerando un caudal de 4 litros por minuto y una tubería de $3 / 8$ de pulgada de diámetro; mediante la Ecuación 1 se establece el área requerida de la tubería.

$$
A=\frac{\pi}{4} \times \theta^{2}
$$

Con la Ecuación 2 se determinó la velocidad que tendría el agua para el caudal antes mencionado.

$$
v=\frac{Q}{A}
$$

Si el objetivo es calentar 4 litros de agua cada minuto se halla que cada litro de agua debe permanecer 15 segundos expuesto al calentador, por lo que se encuentra la longitud de la tubería calefactora con la Ecuación 3.

$$
L=v \times t
$$


A partir de los cálculos se concluyó que se debe tener un serpentín calefactor de al menos $14 \mathrm{~m}$ de largo. De la Ecuación 4 podemos deducir el volumen de agua que se encontrará en el serpentín.

$$
V=A \times L
$$

El siguiente paso es determinar el calor que se requiere transmitir (Ecuación 5).

$$
Q=m \times c \times \Delta T
$$

Donde:

$\mathrm{Q}=$ calor

$\mathrm{m}=$ masa de la sustancia

$\mathrm{c}=$ calor específico del agua

$\Delta \mathrm{T}=$ variación de la temperatura

Con la variación de temperatura deseada, el volumen del agua en el serpentín y la densidad del agua se obtuvo la masa del agua contenida. Finalmente, con la Ecuación 6 se obtiene la potencia requerida para las niquelinas.

$$
P=\frac{Q}{t}
$$

Concluyéndose la necesidad de una potencia mínima de 6500 vatios.

\subsection{Configuración del sensor de caudal}

La configuración del sensor de caudal se lleva a cabo mediante la determinación del número de pulsos que genera este elemento al atravesar un litro de agua por él.

La determinación del caudal se efectúa contando el número de pulsos que genera el sensor en un segundo mediante una interrupción como se muestra en el diagrama de flujo de la Figura 2.

\subsection{Configuración del sensor de temperatura}

La determinación de temperatura a través de un termistor se realiza con la Ecuación 7 que determina la característica específica de este tipo de sensores.

$$
\beta=\frac{\ln \left(\frac{R T 1}{R T 2}\right)}{\frac{1}{T 1}-\frac{1}{T 2}}
$$

Donde:

$\mathrm{T} 1=$ Temperatura en grados kelvin

$\beta=$ Parámetro $\beta$ del sensor

$\mathrm{RT} 1=$ Resistencia del termistor

RT2 = Resistencia de referencia del termistor

$\mathrm{T} 2=$ Temperatura de referencia del termistor
Para encontrar el parámetro beta se hace necesario tener valores de resistencia a dos temperaturas diferentes del termistor y los valores simplemente se deben reemplazar en la Ecuación 7. Para adquirir la señal del termistor se utiliza un divisor de voltaje que indique la variación de temperatura, pero que no linealice el sensor, manteniendo una lectura más fiable.

Posteriormente se realiza la programación misma que permitirá, aplicar los valores que determinen la temperatura real, esto se aprecia en el diagrama de flujo de la Figura 3.

\subsection{Programación del control ON/OFF}

El control ON/OFF se inicializa con una interrupción generada por el pulso que envía un circuito de detección de cruce por cero de la onda de corriente alterna, esta detección inicializa un contador, el mismo que será comparado con una variable ( tencendido $_{\text {) que actúa }}$ como Set Point del sistema y es controlada por un dispositivo externo, esta variable puede adoptar valores que van de 0 a 600 semiciclos de la onda de AC.

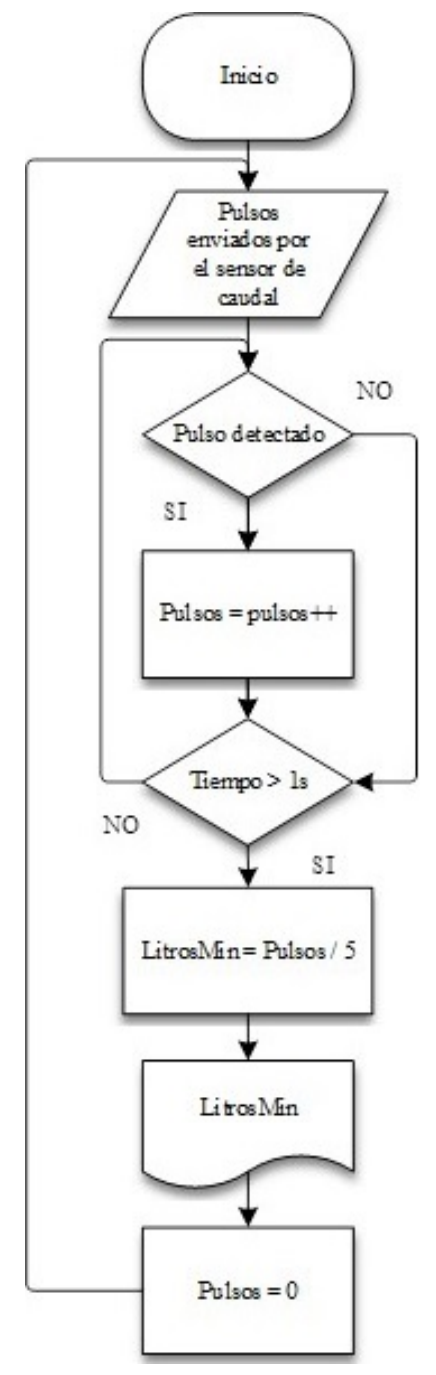

Figura 2. Calibración del sensor de caudal. 
Se usaron valores entre 0 y 600 debido a que en un segundo se producen 120 pulsos y el control está diseñado para 5 segundos de periodo fijo. Si el contador es menor a $t_{\text {encendido }}$ se procede a realizar una nueva comparación la misma que verifica si existe circulación de agua en el sistema. Si son verdaderas las dos comparaciones se encienden los TRIAC (dos por cada fase), caso contrario los TRIAC se desactivan. Finalmente, si el contador es mayor a 600 el mismo se reinicia comenzando de esta manera un nuevo ciclo.

La información generada como la temperatura real y el valor de Set Point son enviados mediante puerto serial a un software diseñado en Matlab para obtener la información que sirva para el modelado del sistema y para el análisis de operación.

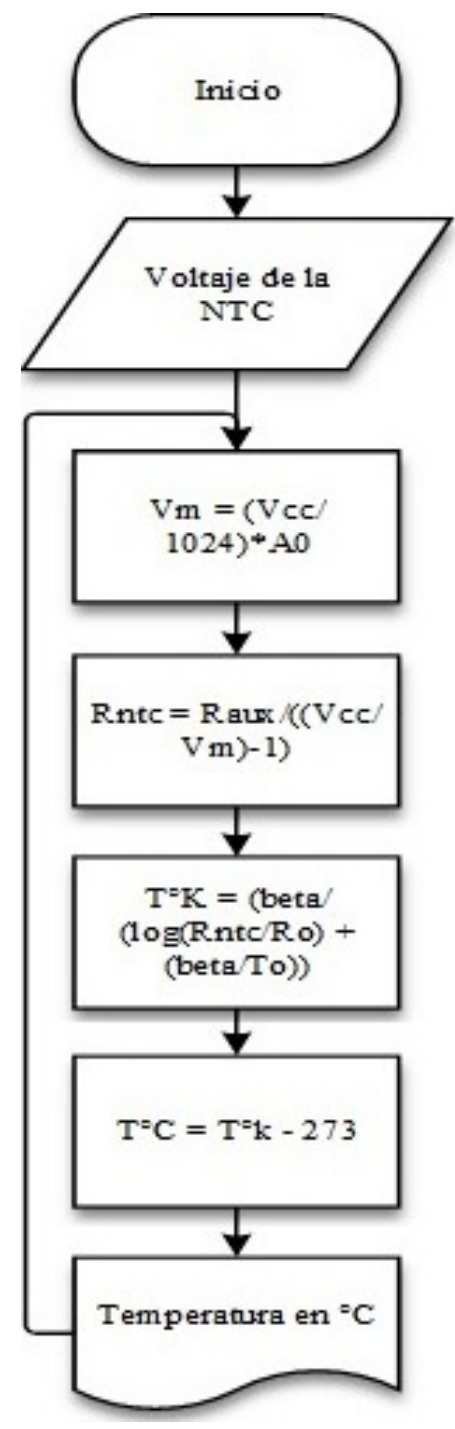

Figura 3. Lectura del sensor de temperatura.

\subsection{Programación del control de fase directa}

En la programación del control de fase directa una vez detectado un pulso generado por el circuito de cruce por cero se inicializa un contador, el mismo que será comparado con una variable de Set Point y que de igual manera es controlada por medio de un dispositivo externo que adopta valores que van de 0 a 180 . Partiendo de que el semiciclo de la onda de corriente alterna se da en un tiempo de 8,33 milisegundos, el valor de 0 en el Set Point representa un tiempo de retardo de $0 \mathrm{~ms}$ para el disparo y un valor de 180 representa un tiempo de retardo de 8,33 ms.

Como el temporizador del microprocesador fue definido a una frecuencia de 46,28 microsegundos, este debe ser multiplicado por valores de 0 a 180 para que de esta manera el contador del temporizador este en el rango de 0 a 8,33 milisegundos respectivamente.

Si el contador es mayor que el Set Point se procede a realizar una nueva comparación la misma que verifica si existe circulación de agua en el sistema. Si son verdaderas las dos comparaciones se encienden los TRIAC y, por tanto, las niquelinas, caso contrario se desactivan.

Finalmente, una vez activado los TRIAC se reinicia el contador esperando ser activado por una nueva interrupción.

\section{Resultados experimentales}

\subsection{Prueba de funcionamiento del control ON/OFF}

Una vez puesto en marcha el controlador ON/OFF, se verificó su funcionamiento normal mediante un osciloscopio en donde se puede apreciar la forma de onda de cómo se encienden y apagan las niquelinas un durante un cierto número de semiciclos de corriente alterna (ver Figura 4).

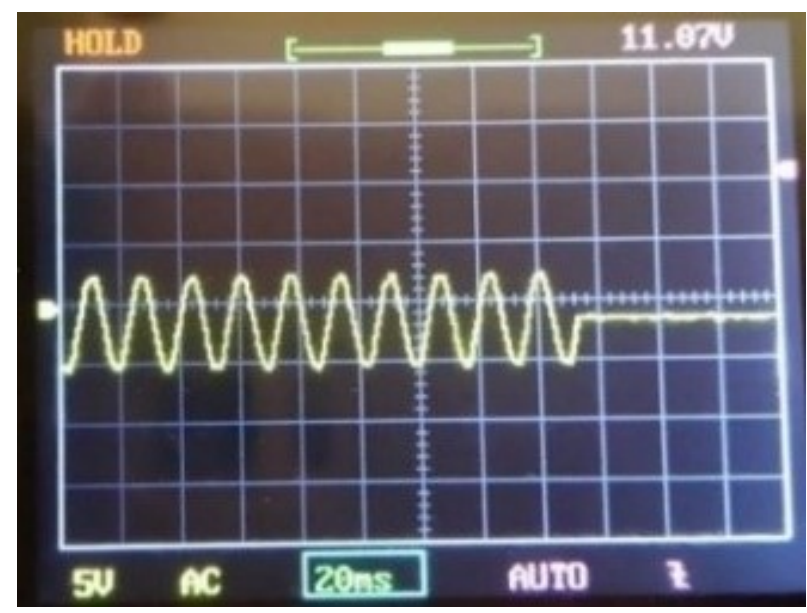

Figura 4. Señal de control ON/OFF. 


\subsection{Prueba de funcionamiento del control de fase directa}

Después de haber realizado la programación del control de ángulo de fase se verificó su correcto funcionamiento observando la forma de onda de voltaje que cae en la carga con la ayuda de un osciloscopio como se ve en la Figura 5, donde se aprecia que la onda de corriente alterna varía su ángulo de encendido.

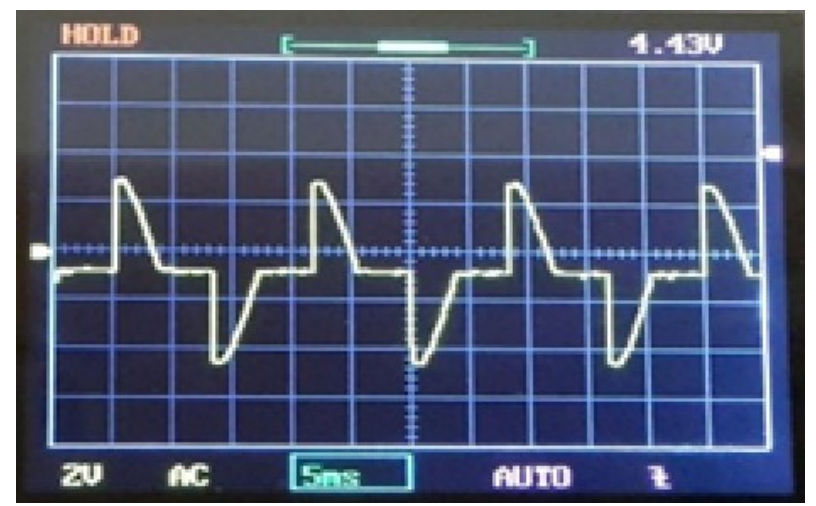

Figura 5. Forma de onda del control de ángulo de fase.

\subsection{PID del control ON/OFF}

\subsubsection{Toma de datos}

Una vez configurado el puerto de comunicación y los límites en el software de Matlab se procede a la toma de datos de temperatura real del sistema y los valores de Set Point durante 17 minutos con 33 segundos obteniendo un total de 30111 datos.

Después de haber terminado el muestreo de datos a diferentes valores de Set Point se obtuvo la gráfica que se muestra en la Figura 6.

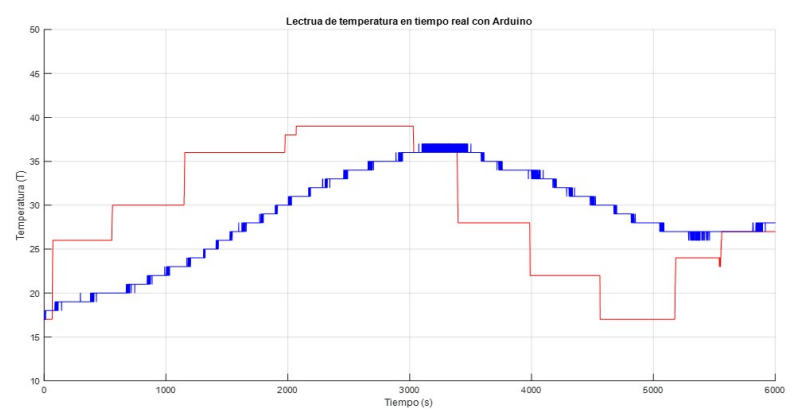

Figura 6. Lectura de temperatura en tiempo real $(\mathrm{ON} / \mathrm{OFF})$.

\subsubsection{Sintonización del control PID}

Con la toma de datos se pudo obtener la función de transferencia del sistema con el tipo de control ON/OFF (Ecuación 8).

$$
F t=\frac{0.0185 s+2.389 e^{-8}}{s^{2}+0.0003364 s+2.087 e^{-8}}
$$

$\mathrm{Y}$ mediante la utilización de la herramienta de sintonización PID Tuner que proporciona Matlab se pudo obtener la respuesta mostrada en la Figura 7, donde se obtiene un estado estable con un tiempo de estabilización excesivamente alto, situación a comprobar en el prototipo físico.

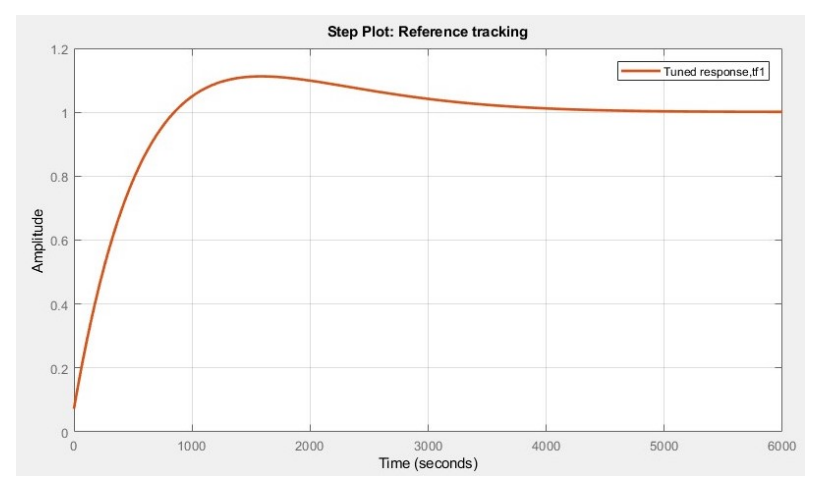

Figura 7. Respuesta de temperatura con el control ON/OFF más un PID.

Una vez realizados los ajustes necesarios en el controlador los parámetros del PID, las constantes se muestran en la Ecuación 9.

$$
\begin{array}{r}
K_{p}=13,534 \\
K_{i}=0,0126 \\
K_{d}=498,9476
\end{array}
$$

\subsection{PID del control de fase directa}

\subsubsection{Toma de datos}

El envío de datos se lo realizó de la misma manera que la utilizada en el control ON/OFF y el tiempo de toma de datos fue de 17 minutos con 47 segundos obteniendo un total de 30523 datos.

Una vez finalizada la toma de información se obtuvo como resultado la gráfica de la Figura 8 y la función de transferencia mostrada en la Ecuación 10.

$$
F t=\frac{0.00016 s+4.58 e^{-7}}{s^{2}+0.002929 s+3.62 e^{-7}}
$$

La función de trasferencia entre un sistema de control y otro no son iguales debido al método que se usa para trasmitir la temperatura al agua.

\subsubsection{Sintonización del control PID}

La sintonización del control PID se realiza de manera similar a la efectuada en el control ON/OFF y se obtuvo la gráfica de la Figura 9 que representa el comportamiento que tendrá el sistema al aplicar este controlador. 


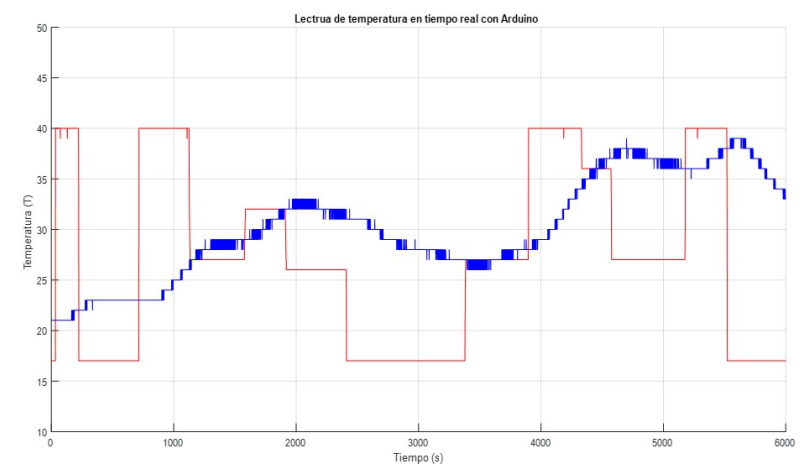

Figura 8. Lectura de temperatura en tiempo real (fase directa).

Después de haber configurado la velocidad de respuesta y la robustez del controlador se obtuvo los parámetros del regulador PID siendo los que se muestran en la Ecuación 11.

$$
\begin{array}{r}
K_{p}=15,8519 \\
K_{i}=0,0126 \\
K_{d}=498,9476
\end{array}
$$

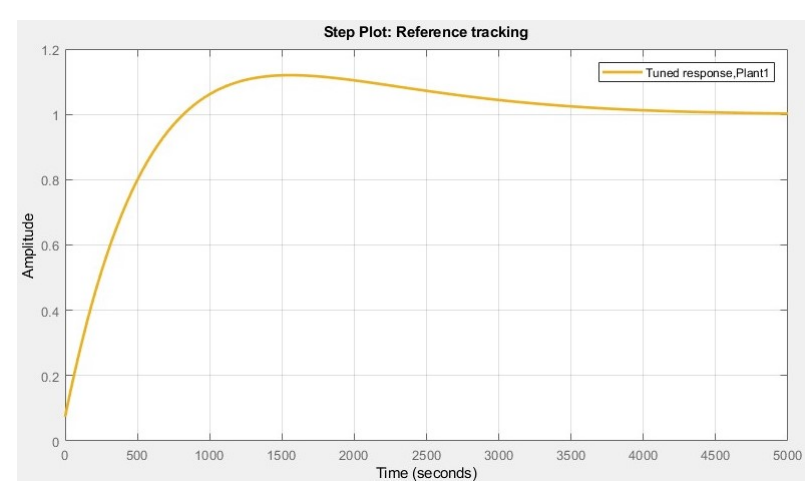

Figura 9. Respuesta de temperatura con el control de fase directa más un PID.

\subsection{Pruebas de funcionamiento de los contro- ladores instalados el PID}

\subsubsection{Pruebas de funcionamiento del control ON/OFF instalado el PID}

En la Figura 10 se observa la respuesta de temperatura del sistema implementado el controlador PID, donde se observa que la temperatura se estabiliza en los valores indicados por el Set Point (línea roja), con un error de menos de un grado centígrado, además, se puede ver que el tiempo de estabilización esta alrededor de unos 500 segundos.

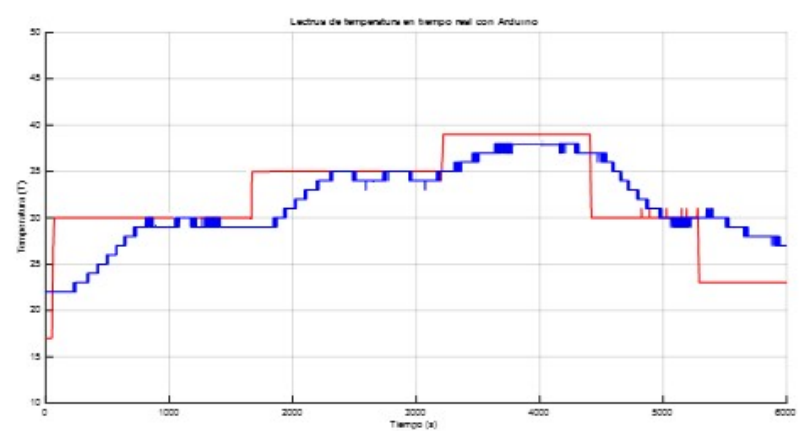

Figura 10. Funcionamiento del control ON/OFF con PID.

\subsubsection{Pruebas de funcionamiento del control por ángulo de fase instalado el PID}

La Figura 11 representa el funcionamiento del control de ángulo de fase una vez instalado el controlador PID, donde se distingue que la temperatura se mantiene estable después de un tiempo de 1000 segundos.

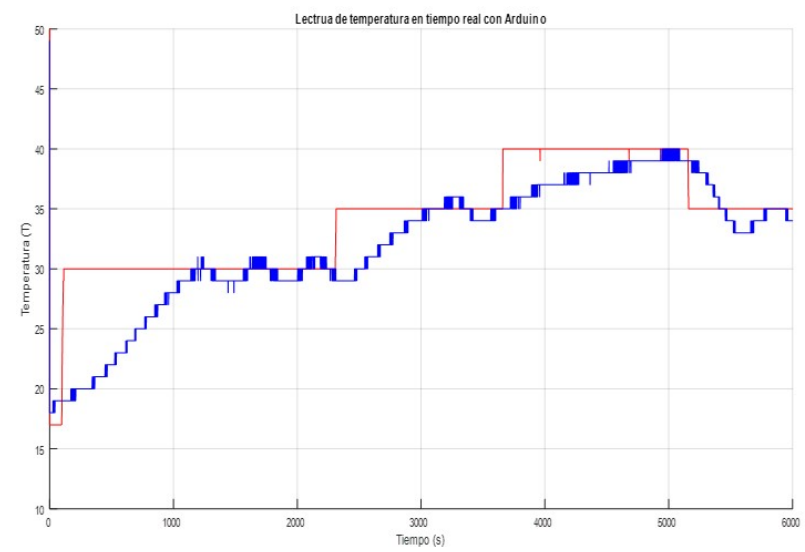

Figura 11. Funcionamiento de fase directa con PID.

\subsection{Comparación de controladores}

\subsubsection{Comparación de eliminación de error entre el control de fase directa y el control ON/OFF instalados los PID}

De los datos obtenidos de pruebas de más de $17 \mathrm{mi}-$ nutos para cada sistema de control se concluye que el control ON/OFF estabiliza la temperatura del agua casi en la mitad de tiempo que el control por ángulo de fase.

Como se observa en las Figuras 10 y 11 al estabilizarse la temperatura, el control de fase directa mantiene de manera más efectiva el valor de temperatura en comparación con el control ON/OFF, es decir, el control de fase directa tiene mayor robustez que el control ON/OFF. 


\subsubsection{Comparación de estabilidad entre el control de fase directa $y$ el control ON/OFF instalados los PID}

La Figura 12 muestra la señal de respuesta en tiempo real de la temperatura del agua utilizando el sistema de control ON/OFF, en ella se aprecia que el sistema de control tarda unos 1000 segundos en calentar el agua hasta unos $38^{\circ} \mathrm{C}$ (temperatura de la prueba) y que una vez alcanzado el valor logra mantener estable la temperatura por el lapso que se realizó la prueba que fue de 90 minutos, salvo una pequeña variación de 2 grados centígrados en los últimos 500 segundos de la prueba.

En la Figura 13 se observa la respuesta de la temperatura del agua con el sistema de control por fase directa, en este caso se observa que el agua alcanza el nivel deseado de temperatura en unos 1100 segundos, casi dos minutos más que el control ON/OFF, y se observa que tiene una variación de $+/-1$ grado centígrado cada 10 segundos. Esto puede deberse a una mala sintonización del regulador PID o a la lentitud de las niquelinas en calentar el agua.

De esta prueba se podría concluir que si bien las respuestas de temperatura son muy similares por estabilidad, robustez y tiempos de estabilización el control ON/OFF resulta más eficiente.

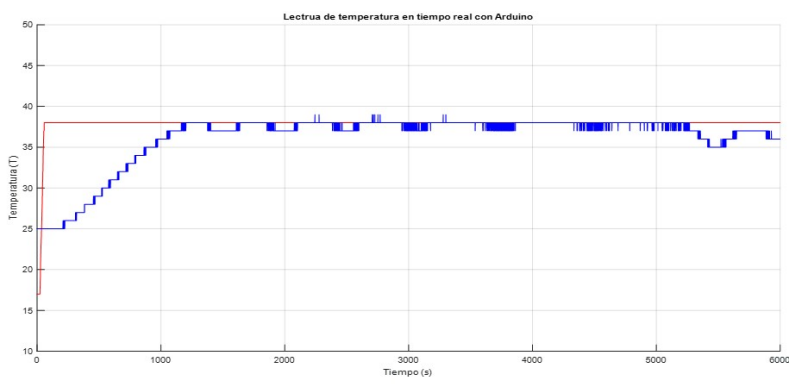

Figura 12. Prueba de estabilidad de temperatura del control ON/OFF más PID.

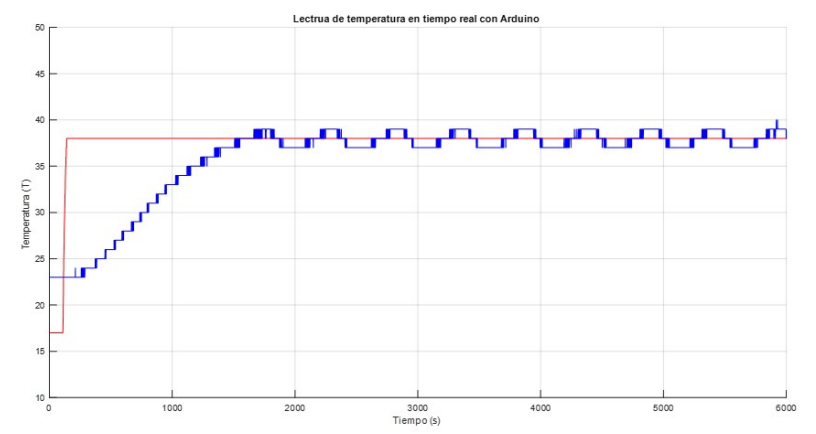

Figura 13. Prueba de estabilidad de temperatura del control por ángulo de fase más PID.

\subsection{Costo de utilización del control de ángulo de fase, el control ON/OFF, la ducha eléctrica y el calefón de gas}

En las pruebas de estabilidad se utilizó cada sistema de control por un lapso de 90 minutos, con ese tiempo de utilización se realizó también la medida del consumo eléctrico de cada controlador, resultando que el controlador ON/OFF consumió $5,45 \mathrm{kWh}$ y el control por ángulo de fase $2,93 \mathrm{kWh}$. Estas medidas se las obtuvo directamente de un medidor de energía.

Tomando en cuenta que al día 5 personas consumen 195 litros de agua y que el prototipo tiene la capacidad de calentar 240 litros en una hora se realizó el cálculo de costos considerando el consumo mencionado anteriormente por unos 22 días al mes que se estimó como promedio del uso del agua para las actividades de aseo personal.

Con estos antecedentes se calculó un costo aproximado de $\$ 8,66$ al utilizar el controlador ON/OFF y un valor de $\$ 4,97$ al utilizar el controlador de fase directa.

La misma proyección se realizó para evaluar el consumo de la ducha eléctrica, donde se estimó la utilización de una ducha de 4500 vatios de potencia, si se repite el ejercicio se determina un consumo de $\$ 8,98$. Mientras que para calentar la misma cantidad de agua con un calefón de gas se necesitará cerca de dos cilindros comerciales ecuatorianos, realizando la proyección de consumo de GLP se calculó un consumo de $\$ 4,59$.

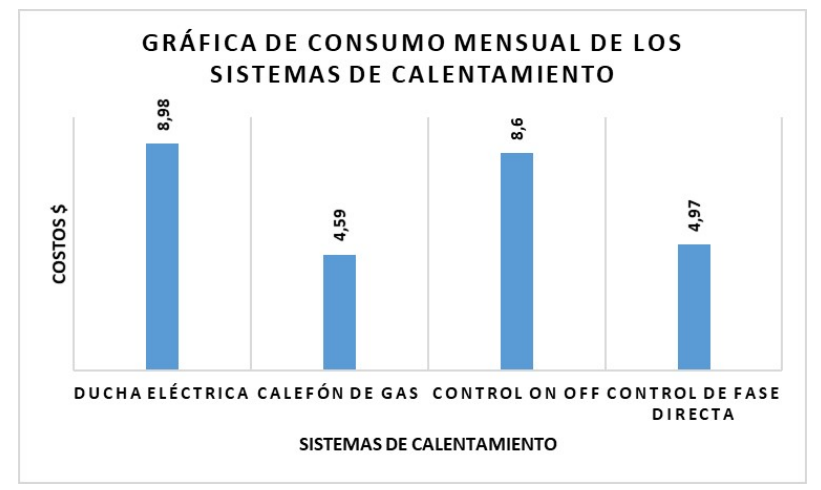

Figura 14. Consumo mensual de los sistemas de calentamiento.

Al revisar los costos de utilizar la ducha eléctrica, el calefón de gas y el control ON/OFF podemos decir que este último consume 38 centavos menos que la ducha eléctrica, pero 4,01 dólares más que el calefón de gas.

Mientras que la utilización del calefón eléctrico con un control de ángulo de fase consume 4 dólares menos que una ducha eléctrica, $\$ 3,63$ menos que el mismo calefón con el control ON/OFF y solo 38 centavos de dólar más que un calefón a GLP. 


\section{Conclusiones}

Mediante la implementación del sistema de calentamiento de agua y al efectuar las pruebas de funcionamiento del mismo se pudo evidenciar que cuatro niquelinas de 1650 vatios cada una y un serpentín de 14 metros de longitud construido con tubería de cobre de $3 / 8$ de pulgada de diámetro es capaz de incrementar la temperatura de 4 litros por minuto de agua desde los 17 hasta los $40^{\circ} \mathrm{C}$ tal y como se había descrito teóricamente en el cálculo de la potencia de las resistencias de calentamiento.

El prototipo funciona con una alimentación bifásica de $220 \mathrm{VAV}$, cada fase de $120 \mathrm{VA}$ alimenta en paralelo a dos niquelinas por intermedios de dos TRIAC, si se tiene en cuenta la potencia de las niquelinas se puede decir que cada elemento de potencia debe soportar al menor una corriente de 14,77 A. Una vez realizadas las pruebas de operación se concluye que los elementos eléctricos y electrónicos del prototipo soportan eficientemente este valor de corriente y se puede operar sin riesgo alguno.

Mediante las pruebas de funcionamiento de los controladores de temperatura se pudo apreciar que el controlador ON/OFF entrega una mejor respuesta en términos de operación que el controlador por fase directa, es decir, el control ON/OFF presenta un menor tiempo de estabilización y una mejor estabilidad en estados estables.

Realizando el análisis de costos se aprecia que el controlador por fase directa presenta un consumo a máxima temperatura de $2,93 \mathrm{kWh}$ y el controlador ON/OFF un consumo de $5,45 \mathrm{kWh}$ igual a máxima temperatura, es decir, en términos energéticos el control por fase directa presenta una gran ventaja sobre el controlador ON/OFF.

Evaluando los beneficios de cada controlador se concluye que a pesar de que el control ON/OFF presenta mejor respuesta en operación, el enorme ahorro del control por fase directa inclina la balanza hacia este para la implementación en trabajos futuros.

Se concluye también la necesidad de probar más técnicas de control, de tal manera que se encuentre el mejor modelo para respuesta de operación y costos, así como se plantea realizar un análisis comparativo para encontrar la afectación que tengan estas distintas técnicas de control sobre la red de distribución eléctrica.

\section{Referencias}

[1] República del Ecuador. (2015) Ley Orgánica de Régimen Tributario Interno (LORTI). Registro Oficial Suplemento 463 de 17 de noviembre de 2004. [Online]. Available: http://bit.ly/2X40v2B
[2] N. Yamamoto, H. Yamaguchi, T. Nagano, M. Higashiuchi, and T. Nanbu, "Gas water heater," United States Patent US D483,451 S, 2003. [Online]. Available: http://bit.ly/2X0UKNY

[3] O. Tsutsui, S. Murakami, H. Kuwahara, and S. Yasunaga, "Instantaneous gas water heater," United States Patent 4,501,261, 1985. [Online]. Available: http://bit.ly/2FGK8yj

[4] T. W. Clifford, "Gas water heater and method of operation," United States Patent US 6,880,493 B2, 2005. [Online]. Available: http://bit.ly/2Yj5bOk

[5] J. H. Brandt, R. T. Meyer, and B. N. Plank, "Control system for a water heater," U.S. Patent 5,797,358, 1998. [Online]. Available: http://bit.ly/2Fvj192

[6] C. Samaniego-Ojeda, O. H. A. Hernández, and J. M. Correa, "Emisiones provocadas por combustión de GLP a partir de calefones en la ciudad de Loja y su posible relación con enfermedades respiratorias agudas (ERA's)," CEDAMAZ, vol. 6, no. 1, pp. 60-67, 2016. [Online]. Available: http://bit.ly/2Ybqvp5

[7] M. Linares, "Contaminación intradomiciliaria," Medwave, vol. 9, no. 1, p. e3697, 2009. [Online]. Available: http://doi.org/10.5867/medwave.2009. 01.3697

[8] E. N. Correa, A. Herrerías, A. Albornoz, G. Villarroel, and A. P. Arena, "Comparación económico-ambiental del uso de energía solar respecto al gas natural para agua caliente sanitaria en la ciudad de Mendoza," Avances en Energías Renovables y Medio Ambiente, vol. 8, no. 1, pp. 01.105-01-110, 2004. [Online]. Available: http://bit.ly/2FreMvq

[9] J. Pesántez, "Reducción de costos en el calentamiento de agua en Ecuador, a través de la sustitución de calefones con uso de GLP por sistemas de energía solar térmica," Revista Científica y Tecnológica UPSE, vol. 1, no. 1, 2012. [Online]. Available: https://doi.org/10.26423/rctu.v1i1.2

[10] E. A. Meléndez Aguilera and H. Soto Nilo, Análisis comparativo, energético y ambiental, en calefones de uso doméstico que operan con gas licuado de petróleo y gas natural. Universidad de Chile, 2007. [Online]. Available: http://bit.ly/2N9UY63

[11] Agencia Estatal Boletín Oficial del Estado. (2019) Real decreto $244 / 2019$, de 5 de abril, por el que se regulan las condiciones administrativas, técnicas y económicas del autoconsumo de energía eléctrica. Ministerio de la Presidencia, Relaciones con las 
Cortes e Igualdad, España. [Online]. Available: http://bit.ly/31QYG7Q

[12] Y. Valdivia Nodal, Y. Díaz Torres, and M. Lapido Rodríguez, "Alternativas de producción de agua caliente sanitaria en instalaciones hoteleras con climatización centralizada," Revista Universidad y Sociedad, vol. 7, pp. 88-94, 122015. [Online]. Available: http://bit.ly/2Y9MRrd

[13] B. Supriyo, Dadi, S. Warjono, A. Wisaksono, S. W. P. Astuti, and K. Utomo, "Pid based air heater controller implemented with Matlab/Simulink and Arduino uno," 2018 5th International Conference on Information Technology, Computer, and Electrical Engineering (ICITACEE), pp. 28-32, 2018. [Online]. Available: https://doi.org/10.1109/icitacee.2018.8576955

[14] S. Lanfredi, R. L. Grosso, A. C. Antunes, S. R. M. Antunes, and M. A. L. Nobre, "Comportamento eléctrico a alta temperatura de termistor cerámico alfa- $\mathrm{Fe}_{2} \mathrm{O}_{3}$ com coeficiente de temperatura negativo," Cerámica, vol. 54, pp. 443-450, 122008. [Online]. Available: http://bit.ly/2J82hpx

[15] M. H. Rashid, Electrónica de potencia: circuitos, dispositivos y aplicaciones. Pearson Education, 2004. [Online]. Available: http://bit.ly/2Kzz75A

[16] M. D. Khairunnas, E. Ariyanto, and S. Prabowo, "Design and implementation of smart bath water heater using Arduino," in 2018 6th International Conference on Information and Communication Technology (ICoICT), May 2018, pp. 184-188. [Online]. Available: https://doi.org/10.1109/ICoICT.2018.8528772

[17] Y. A. Badamasi, "The working principle of an Arduino," in 2014 11th International Conference on Electronics, Computer and Computation (ICECCO), Sep. 2014, pp. 1-4. [Online]. Available: https://doi.org/10.1109/ICECCO.2014.6997578

[18] A. M. Vega E., F. Santamaría P., and E. Rivas T., "Internet de los objetos empleando arduino para la gestión eléctrica domiciliaria," Revista $E A N$, pp. 23-41, 07 2014. [Online]. Available: http://bit.ly/2RB2sxw

[19] A. L. Silva, M. Varanis, A. G. Mereles, C. Oliveira, and J. M. Balthazar, "A study of strain and deformation measurement using the Arduino microcontroller and strain gauges devices," Revista Brasileira de Ensino de Física, vol. 41, no. 3, pp. e20 180 206-1-e20180 206-7, 002019. [Online]. Available: http://dx.doi.org/10.1590/ 1806-9126-RBEF-2018-0206

[20] A. A. Custodio Ruiz and R. Torres, "Conexión directa de múltiples sensores a microcontroladores sin utilizar convertidor analógico digital," Universidad, Ciencia y Tecnología, vol. 10, no. 39, pp. 147-151, 07 2006. [Online]. Available: http://bit.ly/2J2ig8n

[21] P. H. Guadagnini and V. E. Barlette, "Um termômetro eletrónico de leitura direta com termistor," Revista Brasileira de Ensino de Física, vol. 27, no. 3, pp. 369-375, 09 2005. [Online]. Available: http: //dx.doi.org/10.1590/S1806-11172005000300011

[22] H. Bory Prevez, H. Martínez García, L. Vázquez Seisdedos, F. Chang Mumañ, and L. A. Enríquez García, "Comparación entre rectificador trifásico con conmutación simétrica y convertidor ac/ac para la mejora del factor de potencia en microcentrales hidroeléctricas," Revista Iberoamericana de Automática e Informática industrial, vol. 15, no. 1, pp. 101-111, 2017. [Online]. Available: https://doi.org/10.4995/riai.2017.8816

[23] C. Scianna, "Dimming circuit for led lighting device with means for holding triac in conduction," Unioted States Patent WO 2005/115058 A1, 2005. [Online]. Available: http://bit.ly/2X0f3Lx

[24] A. Dytch, M. Lane, A. Keatley, and W. Wright, "Microprocessor controlled through-flow electric water heater," U.S. Patent 4,638,147, 1987.

[25] I. A. Ruge Ruge, "Optimización de señal de control en reguladores PID con arquitectura antireset Wind-Up," Tecnura, vol. 15, no. 30, pp. 24-31, 12 2011. [Online]. Available: http://bit.ly/2FxFNNM

[26] L. F. Lozano-Valencia, L. F. RodríguezGarcía, and D. Giraldo-Buitrago, "Diseño, implementación y validación de un controlador PID autosintonizado," TecnoLógicas, no. 28, pp. 33-53, 06 2012. [Online]. Available: http://bit.ly/2WXkb3f 